\title{
Phase Diagram of Spinless Fermions on an Anisotropic Triangular Lattice at Half-filling
}

\author{
Chisa Hotta *, Nobuo Furukawa, Akihiko Nakagawa, Kenn Kubo \\ Aoyama Gakuin University, Fuchinobe 5-10-1, Sagamihara-shi, Kanagawa 229-8558, Japan
}

(Received September 28, 2018)

The strong coupling phase diagram of the spinless fermions on the anisotropic triangular lattice at half-filling is presented. The geometry of inter-site Coulomb interactions rules the phase diagram. Unconventional charge ordered phases are detected which are the recently reported pinball liquid and the striped chains. Both are induced by the quantum dynamics out of classical disordered states and afford extremely correlated metallic states and the particular domain wall-type of excitations, respectively. The disorder once killed by the quantum effect revives at the finite temperature, which is discussed in the terms of the organic $\theta-\mathrm{ET}_{2} X$.

KEYWORDS:

Charge degrees of freedom provide particular orders and disorders under geometrical frustration, as has been discussed from the time of the Verwey transitions. ${ }^{1}$ Many recent topics are related to those on the anisotropic triangular lattice(ATL). They are experimentally explored in the organic solids as a possible "non-magnetic" Mott insulator $^{2}$ and organic thyristor. ${ }^{3}$ Theoretically, the phase diagram of the half-filled Hubbard model on ATL seems to suggest the particular "spin liquid" insulator, ${ }^{4}$ which has been searched for from the past as a nonmagnetic state free from any kind of ordering. ${ }^{5}$

Quarter-filling is characterized by the charge ordering $(\mathrm{CO})$ phenomenon, as studied by the extended Hubbard model(EHM) in the context of organic solids. ${ }^{6,7}$ The interplay of $\mathrm{CO}$ and frustration is investigated in the phase diagrams based on ATL. ${ }^{8,9}$ However, the two dimensional systems under geometrical frustration requires a delicate and difficult numerical treatment, thus a clear answer is still not obtained. In the present letter, we simplify the problem and deal with the spinless fermions at half-filling to extract the intrinsic character of the charge degrees of freedom. We obtain a convincing phase diagram which retains a strong coupling nature even in a rather weak coupling region. In addition to the pinball liquid reported in Ref.[10], another unconventional strongly correlated $\mathrm{CO}$ state with fluctuating stripes is found in the finite size cluster. Its transport character is sensitive to the geometry of quantum dynamics which is discussed in terms of the organic solid $\theta-\mathrm{ET}_{2} X$.

The spinless fermion Hamiltonian in the present study is given as,

$$
\mathcal{H}=\sum_{\langle i, j\rangle}\left(-t_{i j} c_{i}^{\dagger} c_{j}+\text { h.c. }+V_{i j} n_{i} n_{j}\right) .
$$

Here, $c_{j}$ and $n_{j}$ denote the annihilation and number operator of fermions. The triangular lattice has anisotropy in one of three directions as reflected in the transfer integrals, $t_{i j}=t, t^{\prime}$, and the inter-site Coulomb interactions, $V_{i j}=V, V^{\prime}$, between nearest neighbor sites whose corresponding bond directions are defined in Fig.1. The phe-

*E-mail address:chisa@phys.aoyama.ac.jp nomena realized in EHM in the temperature range of $J \sim \frac{t^{2}}{U}<T \ll V$ are suitably described by the $t$ - $V$ model.

We perform the exact diagonalization on $N=4 \times 6=$ 24 cluster in eq.(1). Figure 1 shows the phase diagram on the plane of $V / t$ and $V^{\prime} / t$ at several fixed values of $t^{\prime} / t$ with periodic boundaries. The diagram is classified into three different regions, whose boundaries are estimated from the discontinuity in charge structural factor, the gradient of energy by $V$ and $V^{\prime}$, and Drude-like component as we see shortly. The phases (I) and (III) are the two-fold periodic striped CO states whereas the phase (II) has a three fold periodicity and is denoted as "a pinball liquid" which is studied in Ref.[10]. The $t^{\prime} / t$ dependence of the phase boundaries is very small. This phase diagram describes well the strong coupling physics at around $V, V^{\prime}>2 t$ below which the phase transition begins to smear.

We first present the structural factor, $C_{k}=$ $\frac{1}{N} \sum_{l m}\left\langle\left(n_{l}-1 / 2\right)\left(n_{m}-1 / 2\right)\right\rangle \mathrm{e}^{\mathrm{i}\left(r_{l}-r_{m}\right) k}$, in Fig. 2. Each phase is characterized by its own wave numbers, (I) $\left(\frac{\pi}{2} n, \pi\right)$ with integer $n$, (II) $\left(\pi, \pm \frac{2}{3} \pi\right),\left(0, \pm \frac{4}{3} \pi\right)$, and (III) $(\pi, 0)$. The peak amplitudes vary discontinuously from one to another at the phase transitions, which confirm their first order character. We mention that the structural factors over the entire Brillouin zone beside each characteristic ones are almost completely suppressed, which means that there is little possibility of the coexistence phase. The amplitudes in (I) and (II) are significantly smaller than the one in (III). As we see shortly, the disorder exists in the classical limit of phases (I) and (II), which still influences the states at $t \neq 0$ and suppresses the two-body correlation.

Figure3(a) shows the ground state energy, $E_{Q}$, which behaves almost linearly with $V$ at the fixed value of $V+V^{\prime} \equiv 2 V_{0}$, while its gradient changes discontinuously at the phase transition. It is also seen in Fig.3(b). Therefore the phase transitions are of first order. Note that the present phase diagram is almost free from finite size effect. Figure 3(a) reveals that $E_{Q}$ of the smaller cluster size, $4 \times 4$ and $4 \times 3$, reproduces within $0.3 \%$ the ones of $4 \times 6$ in (I), (III) and (II), (III), respectively, i.e. as long as the periodicity of each state is commensurate 


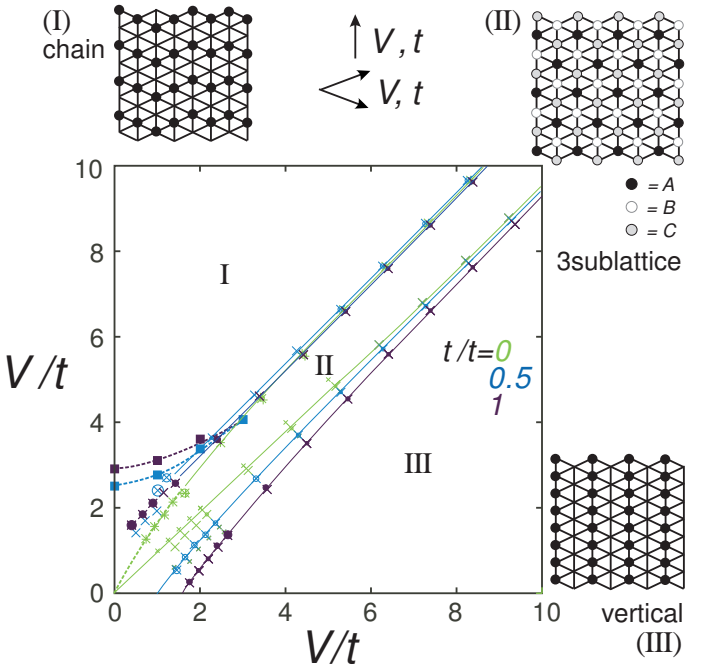

Fig. 1. Ground state phase diagram of eq.(1) on the plane of $V / t$ and $V^{\prime} / t$ obtained numerically at $N=24$. It is classified into three regions, (I) chain stripe, (II) pinball liquid and (III) vertical stripe. The phase boundary is given at $t^{\prime} / t=1,0.5$ and 0 . Broken lines with square symbols indicate the upper bound of the insulating state in (I) for $t / t^{\prime}=0.5,1$. Shaded region includes the possible location of $\theta-\mathrm{ET}_{2} X$. Representative configuration of the classical limit of the three phases are schematically shown together. The arrows with $V, t$ and $V^{\prime}, t^{\prime}$ at the top indicate the directions of interacting bonds in eq.(1).

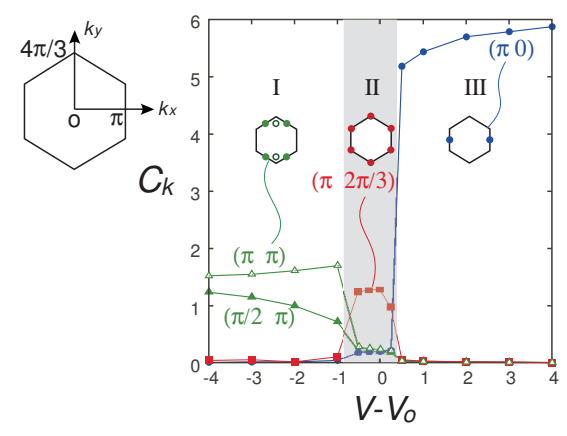

Fig. 2. Amplitude of charge structural factors along $V+V^{\prime}=$ $2 V_{o}=12$ with characteristic wave numbers. Rectangle represents the Brillouin zone and the circles are the location of wave numbers with peaks.

with the shape of the cluster.

The difference between three phases can be clearly understood by the strong coupling theory. ${ }^{10}$ In the classical limit $t=t^{\prime}=0$, the ratio of $V$ and $V^{\prime}$ determines the phase diagram. The system has a semimacroscopically $\left(\sim 2^{\sqrt{N}}\right.$-fold) degenerate chain CO state and a nondegenerate vertical stripe $\mathrm{CO}$ state at $V\left\langle V^{\prime}\right.$ and $\left.V\right\rangle$ $V^{\prime}$, respectively, ${ }^{10}$ and the phases (I) and (III) are their extensions. Their lowest order energy gain per site by $t, t^{\prime}$ $\left(\ll V, V^{\prime}\right)$ is estimated as, $E_{2}=-t^{\prime 2} / V^{\prime}-t^{2} /\left(2 V^{\prime}-V\right)$ (I), $-2 t^{2} /\left(3 V-2 V^{\prime}\right)$ (II), which is presented numerically in Fig. 3(a) as $\left(E_{Q}-E_{C}\right) / N$. Since this effect is small at $V, V^{\prime} \gg t, t^{\prime}$, the stripes are quite robust even though the charge localization length is somewhat extended.

When $V=V^{\prime}$ the three sublattice state with a macroscopic degeneracy joins the ground state besides these
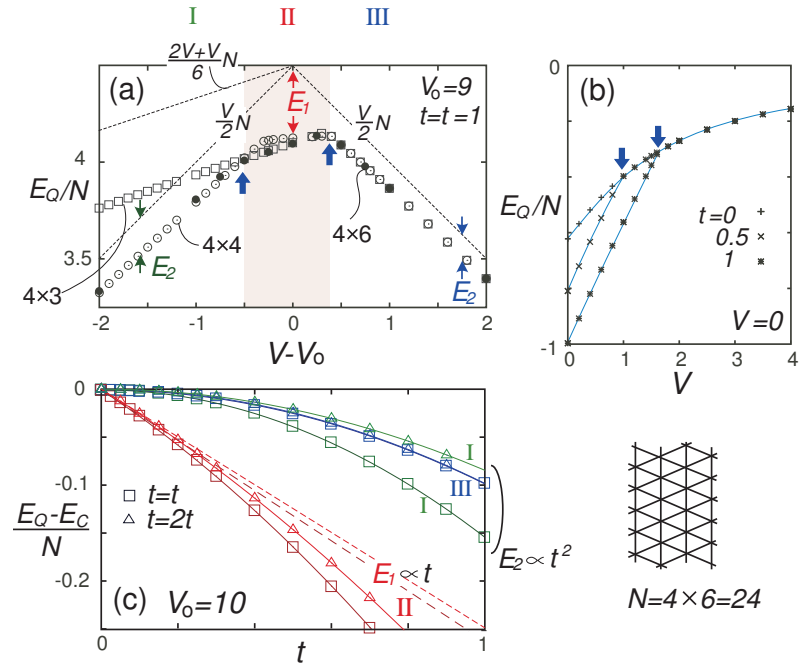

$N=4 \times 6=24$

Fig. 3. Ground state energy of eq.(1), $E_{Q}$, (a) at fixed $V+V^{\prime}=$ $2 V_{o}=18$ with $t=t^{\prime}=1$ under several cluster size. Broken lines give the classical binding energy per site, where $E_{1}, E_{2}$ are the energy gain per site due to $t, t^{\prime}$. The $4 \times 6$ cluster shown together on the right. (b) $V^{\prime}$-dependence of $E_{Q}$ at $V=0$. for several choices of $t^{\prime}=0,0.5,1(t=1)$. Arrows indicate the phase transitions between (I) and (II). (c) Energy correction, $\left(E_{Q}-E_{C}\right) / N$, due to $t=t^{\prime}$ and $t=2 t^{\prime}$ at $V_{o}=20$ in three phases, $V=8(\mathrm{I}), 10(\mathrm{II})$, and $12(\mathrm{III})$, which are assigned $E_{2}, E_{1}$, and $E_{2}$, respectively.

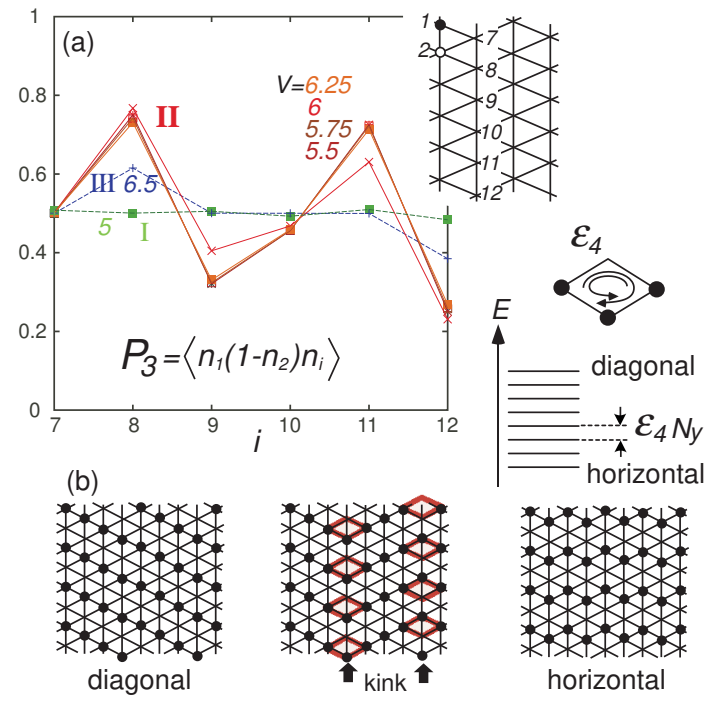

Fig. 4. (a) Three-body charge correlation function, $P_{3}(j)$, along the second $\operatorname{chain}(i=7-12)$ at $t=t^{\prime}=1$ for several choices of $V$ with $V_{o}=6$, with $i$ denotes the site index. Those in broken lines are the cases of striped states. (b) Fourth order process that lifts the degeneracy of the chain state. The cyclic permutation of three charges on the rectangle gives $\epsilon_{4}$. The energy spectrum becomes equally spaced by $\epsilon_{4} N_{y}$, with bottom and top as the horizontal and diagonal $\mathrm{CO}$, respectively. The number kinks including $N_{y^{-}}$ rectangles in bold lines determines the energy.

two stripes in the classical limit. The quantum dynamics of $t$ by first order lifts the degeneracy of part of the three sublattice states and the pinball liquid (II) appears. The estimated kinetic energy gain $\left(\left(E_{Q}-E_{C}\right) / N\right.$ in Fig. 3(a) $)$ is of first order ( $t$-linear) which behaves as $E_{1} \propto c_{1} t+c_{2} t^{2}$ $\left(c_{1}, c_{2}\right.$ are constants) in contrast to $E_{2} \propto t^{2}$ as shown 
J. Phys. Soc. Jpn.

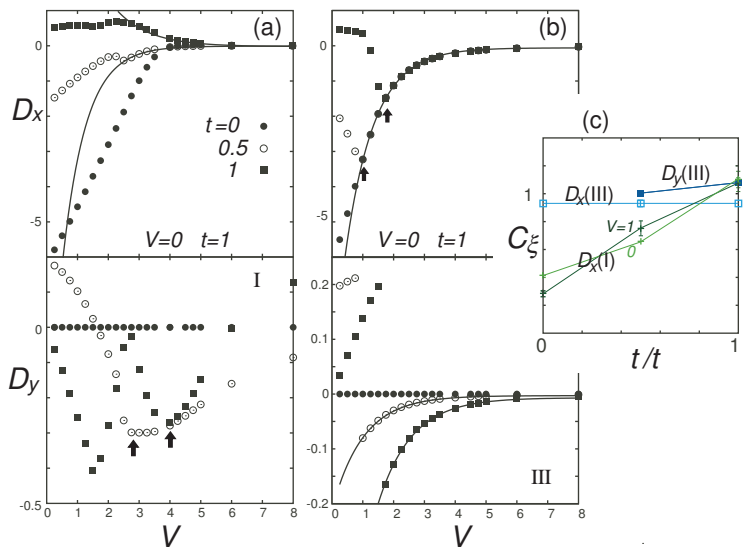

Fig. 5. Amplitude of energy, $D_{\alpha}$, under the twisted boundary condition in $\alpha=x, y$, directions along (a) $V=0$ and (b) $V^{\prime}=0$. Solid lines show the exponential fit, $D_{\alpha} \propto \mathrm{e}^{-\Delta_{c} / C_{\xi_{\alpha}}}$. Arrows indicate the phase transitions (those in (a) are the possible metalinsulator transition points). The corresponding fitting constants, $C_{\xi_{\alpha}}$, are given in (c) as functions of $t^{\prime} / t$

in Fig. 3(c) regardless of the anisotropy in $t^{\prime} / t$. Since $E_{Q}=V / 2+E_{2},\left(2 V^{\prime}+V\right) / 6+E_{1}$, and $V / 2+E_{2}$ for (I)-(III), respectively, the phase (II) appears at around $6\left(E_{1}-E_{2}\right) \leq V-V^{\prime} \leq-3\left(E_{1}-E_{2}\right)$.

To fully figure out the nature of the pinball liquid (II) out of disorder, we should consider the three-body correlation, $P_{3}(j) \equiv\left\langle n_{1}\left(1-n_{2}\right) n_{j}\right\rangle$ which is the population of the $j$-th site when the 1 -st and the 2-nd site is present and absent, respectively. Figure 4(a) shows that the $n_{A}+n_{B} \sim 2 n_{C}$ type of three-fold structure is present only in (II). This is in contrast to the $n_{A} \neq n_{B} \sim n_{C}$ type ones based on mean field wave functions. ${ }^{8}$

The phase (I) is another ordered state out of disorder. Its classical limit has a $\mathrm{CO}$ along every chain ( $y$ direction). Regular stacking of the $\mathrm{CO}$ chains give the diagonal striped structure shown in Fig. 4(b). Starting from this we can introduce a kink one by one, and finally we obtain the horizontal stripe with the full number of kinks. All these structures have the same energy and are semi-macroscopically degenerate. We call this gapless and disordered state a chain CO. ${ }^{10}$ The energy correction by $t$ starts from the second order, but the degeneracy is not lifted until the fourth order; the degree of lifting per rectangular unit with three charges is $\epsilon_{4}=-2 t^{4} /\left(2 V^{\prime}-V\right)^{2} V^{\prime}$ as shown in Fig. 4(b). The energy gain from the diagonal stripe is given as $\epsilon_{4} N_{y} N_{\text {kink }}$, where $N_{\text {kink }}$ denotes the number of kinks. Therefore the horizontal stripe becomes a unique ground state in the bulk limit, and the reduction of $N_{\text {kink }}$ corresponds to the gapful domain wall excitation which requires energy larger than the single particle excitation $\left(\epsilon_{4} N_{y} \gg V^{\prime}\right)$ except in the vicinity of the strong coupling limit. In finite clusters, however, these kinked chain states easily mix with the horizontal stripe. The energy gain here is, $\epsilon_{\mathrm{ch}} \sim N_{y} t^{t^{\frac{N}{2}}} / V^{\prime \frac{N_{y}}{2}-1}$, which is far smaller than $E_{1}$ and $E_{2}$ at $V^{\prime} \gg t^{\prime}$ so that its correction to the phase boundary in Fig. 1 is negligibly small. In this way, the degeneracy lifted ground state in the bulk limit should have only a single peak of $C_{k}$ within the Brillouin zone at $(0, \pi)$.
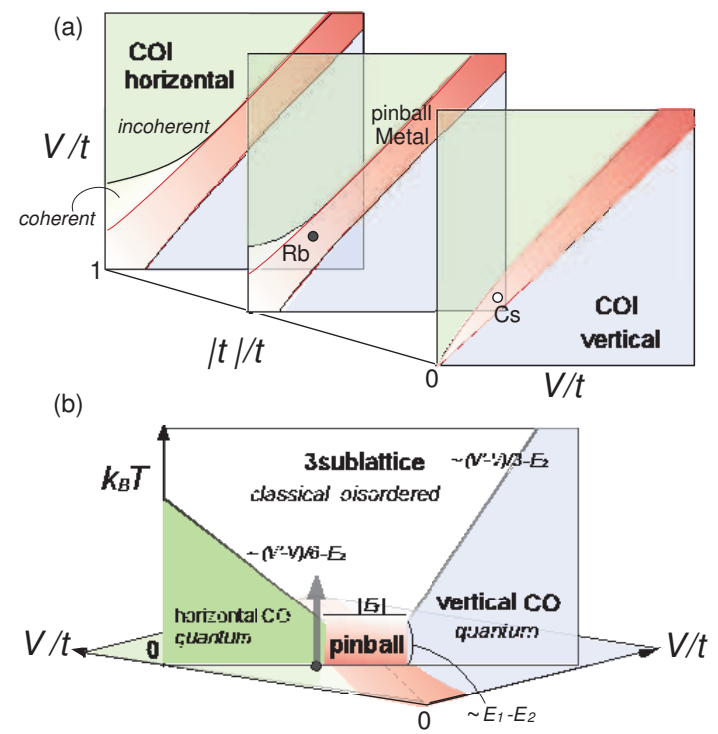

Fig. 6. (a) Schematic ground state phase diagram on the planes of $V / t$ and $V^{\prime} / t$ at each $t^{\prime} / t$. The COI denote the insulating striped phases. Filled and open circles are the possible locations of the Rb and Cs-salts of $\theta-\mathrm{ET}_{2} X$. (b) Schematic phase diagram at finite temperature. The colored regions denote the quantum phases, (I)-(III), which are replaced by the classical disordered phase at higher temperatures. The crossover is possibly realized in the Cs-salt at $T_{\mathrm{cr}}$ as indicated in arrows.

Next, we twist the boundary condition in $x$ and $y$ directions by the phase factor of $\mathrm{e}^{\mathrm{i} \psi_{\alpha}}(\alpha=x, y)$, where $\psi_{\alpha}=0$ and $\pi$ correspond to the periodic and anti-periodic boundary conditions, respectively. Then, $D_{\alpha}=E_{Q}\left(\psi_{\alpha}=\right.$ $\pi)-E_{Q}\left(\psi_{\alpha}=0\right)$, is approximately proportional to the Drude weight of the finite system size. The discontinuity of $D_{\alpha}$ detects the first order phase transitions among (I) (II) and (III). The pinball liquid is possibly a metal in the bulk limit with $D_{\alpha} \neq 0$ as previously reported in Ref.[10] ${ }^{10}$ In contrast, the coherence of the gapful localized commensurate stripes is expected to decay exponentially with distance $l$ as, $\propto \mathrm{e}^{-l / \xi}$, where $\xi$ denotes the coherence length. Then we except, $\xi_{\alpha}=C_{\xi_{\alpha}} / \Delta_{c}$ with $C_{\xi_{\alpha}}$ as $V, V^{\prime}$-independent but $t^{\prime}, t$-dependent values and $\Delta_{c}$ the value of charge gap in the classical limit, i.e. $V^{\prime}$ in (II) and $2 V-V^{\prime}$ in (III). By anticipating $D_{\alpha} \propto \mathrm{e}^{-N_{\alpha} C_{\xi_{\alpha}} / \Delta_{c}}$ ( $N_{\alpha}$ is the system length), the fitting is well performed as the solid lines in Figs. 5(a) and 5(b) whose $C_{\xi_{\alpha}}$ 's are plotted against $t^{\prime} / t$ in Fig. 5(c). The fitting breaks down simultaneously at the transition from (III) to (II), which indicates that phase (III) is a localized $\left(\xi_{\alpha}<N_{\alpha}\right)$ insulator. In this phase both $D_{\alpha}$ and $C_{\xi_{\alpha}}$ are $t^{\prime} / t$-independent which is also the case with $E_{Q}$ in Fig. 3(b). In contrast, $E_{Q}$ of (I) in Fig. 3(d) together with $D_{\alpha}$ actually has large $t^{\prime} / t$-dependence. There is a possible phase boundary within phase (I) estimated by the discontinuity in $\partial D_{\alpha} / \partial V^{\prime}$ which is given in Fig. 1 with broken lines. The strong coupling large $V^{\prime}$-region is an insulator in its bulk limit. However, in the rather weak coupling region, $\xi_{\alpha}$ develops and exceeds $N_{\alpha}$, which corresponds to the above phase boundary. Notice that it depends much on $t^{\prime} / t$. At present, we cannot discriminate whether this new intermediate region in (I) is gapful or not in the bulk case, nor 
whether there is a finite size effect in this boundary. Nevertheless, the estimated boundary gives an upper bound of the metal insulator transition line.

The previous theoretical attempts to clarify the phase diagram suffers from problems. Those based on meanfield wave function ${ }^{8}$ failed to describe fully the extremely correlated chain(horizontal) and pinball states. Their phase diagram has ambiguous "competition" region between states of different symmetry breaking due to large size-dependence, which is an artifact of adopting a plane wave based wave function on such correlated and highly localized system. Exact diagonalization on EHM in $4 \times 4$ finite clusters overestimates their "frustration induced metal" as well as underestimates the pinball liquid, ${ }^{9}$ as clarified explicitly in Figs. 1 and 3(a). Our phase diagram presents a clear view to these problems and describes the intrinsic nature of $U=\infty \mathrm{EHM}$ at quarter-filling since the basic character of three phases are free from statistics. ${ }^{10}$

The ground state phase diagram is summarized in Fig. 6(a). The anisotropy of $t^{\prime} / t$ only affects the coherence of phase (I) in a relatively weakly coupled region. We show schematically in Fig. 6(b) the finite temperature effect, $k_{B} T \neq 0$, where the classical disordered state becomes prosperous as a result of the macroscopic entropy. The free energy of the classical three-sublattice state is, $F_{3 \mathrm{sub}} \sim \frac{2 V+V^{\prime}}{6} N-k_{B} T \ln \left(0.5 \times 2^{N}\right)$, while those of the nondegenerate quantum states are $F=E_{Q} \sim$ $E_{C}+\delta E_{Q} N$ with $\delta E_{Q} \sim E_{2}+\epsilon_{4}, E_{1}$, and $E_{2}$ for (I), (II) and (III), respectively. The crossover lines from quantum to classical states with increasing $k_{B} T$ are estimated by the crossing points of these free energies as, $T_{\text {cr }} \sim \frac{V^{\prime}-V}{6}-E_{2},\left|E_{1}\right|$ and $\frac{V-V^{\prime}}{3}-E_{2}$, respectively. The chain type of disorder does not appear in the bulk case since the entropy gain remains of order $\sim O(\sqrt{N} \ln \sqrt{N})$.

Now we examine the relevance of the present results to the $\mathrm{CO}$ materials, $\theta-\mathrm{ET}_{2} X$. The nature of this family at low temperatures is classified on the experimental phase diagram along the dihedral angle, $\phi ;^{12}$ when $\phi>110^{\circ}$, the system becomes a CO insulator accompanied by the structural transition at $T_{\mathrm{CO}} \sim 200 \mathrm{~K}$. At $\phi<110^{\circ}$ it is a bad metal down to low temperatures with the resistivity minimum at $T_{\rho} \sim 20-70 \mathrm{~K}$. Recent $\mathrm{X}$-ray analysis reports the existence of a short range diffuse spot of two-fold and three-fold type in the Cssalt $\left(X=\mathrm{Cs} M(\mathrm{SCN})_{4}, M=\mathrm{Co}, \mathrm{Zn}, \phi<110^{\circ}\right)$ at $\sim T_{\rho}{ }^{13}$ and also in Rb-salts $\left(\phi \sim 110^{\circ}\right)$ above $T_{\mathrm{CO}},{ }^{14}$ which have $\left(\left|t^{\prime}\right| / t, V^{\prime} / V\right) \sim(0,0.9)$ and $(0.5,0.85)$, respectively. ${ }^{15}$

It is obvious that there is a characteristic crossover temperature of order $T_{\rho}$ at $\phi<110^{\circ}$ which increases with $\phi$. Actually, at around $V^{\prime}>V$, we can estimate the crossover temperature as $T_{\mathrm{cr}} \sim \frac{V^{\prime}-V}{6} \sim 0.1 V$. Since we consider $V \sim T_{\mathrm{CO}}$, the experimental crossover at $\sim T_{\rho}$ is reasonably understood. An observed bad metallic nature with almost $T$-independent resistivity at $T>T_{\rho}{ }^{12}$ supports the existence of disorder. Also the up going of resistivity at $T<T_{\rho}$ is consistent with the insulating character of the possible horizontal stripe. ${ }^{12}$ The coexistence of states with different periodicity in the ground state is ruled out in the present study. However, at finite temperature, the disordered state which includes both the three-sublattice and the striped local patterns might appear around the crossover temperature, which were excluded in Fig. 6(b) for simplicity. The study on this complicated matter is still underway.

Somehow, the ground state of this family is placed amidst the pinball liquid state as shown in Fig. 1, which is inconsistent with the above experimental findings. In the present stage we did not consider the effect of phonons. The three-fold state is found to be quite easily replaced by the horizontal stripe long range order(LRO) under that effect, ${ }^{17}$ which might be valid in our case as well.

It is frequently argued that $\phi$ can be regarded as $V / t$. Contrarily, one of the authors successfully reclassified the experimental phase diagram by $\left|t^{\prime}\right| / t$, on the basis of $\mathrm{EHM}^{7}$ which is recently pursued experimentally ${ }^{16}$ and also in the weak coupling theory. ${ }^{8}$ In our phase diagram, however, $\left|t^{\prime}\right| / t$ only rules the coherence length of the system at the relatively weak coupling region. To figure this out, we should examine the extra effect such as electronphonon coupling in the disordered region.

In conclusion, the strong coupling phase diagram of the charges on the anisotropic triangular lattice is presented. A metallic pinball liquid phase appears in between an insulating vertical and the horizontal stripe CO's. The first order transitions take place in the phase boundaries which are almost solely determined by the geometry of the inter-site Coulomb interactions. The present phase diagram provides two different types of order-from-disorder due to quantum dynamics; the extremely correlated pinball liquid out of disordered three sublattice classical state, and the horizontal state which has a domain-wall like excitation to the disordered chain striped classical states. Both of these states provide good test cases for the disorder-embedded phenomena. The disorder once gave path to quantum states recovers at finite temperature with the aid of its macroscopic entropy.

We thank R. Kondo, M. Watanabe, I. Terasaki, and R. Chiba for many useful experimental informations.

1) E. J. W. Verwey, Nature 144327 (1939).

2) Y.Shimizu, K. Miyagawa, K. Kanoda, M Maesato and G. Saito, Phys. Rev. Lett. 91 (2003)107001.

3) F. Sawano, I. Terasaki, H. Mori, T. Mori, M. Watanabe, N. Ikeda, Y. Nogami, Y. Noda, Nature 437 (2005) 522.

4) H. Morita, S. Watanabe and M. Imada, J. Phys. Soc. Jpn. 71 (2002) 2109.

5) P. W. Anderson, Mater. Res. Bull. 8 (1973) 153; P. Fazekas and P. W. Anderson, Philos. Mag. 30 (1974) 423.

6) H. Seo, C. Hotta, H. Fukuyama, Chem. Rev. 104 (2004) 8558, and the references therein.

7) C. Hotta, J. Phys. Soc. Jpn. 72 (2003) 840

8) H. Watanabe, M. Ogata, J. Phys. Soc. Jpn. 75 (2006) 063702.

9) J. Merino, H. Seo, M. Ogata, Phys. Rev. B 71 (2005) 125111.

10) C. Hotta, N. Furukawa, condmat/0605045/.

11) G. H. Wannier, Phys. Rev. 79 (1950) 357; K. Husimi, I. Shoji, Prog. Theor. Phys. 5 (1995) 341, and so on.

12) H. Mori, S. Tanaka, and T. Mori, Phys. Rev. B 57 (1998) 12023.

13) M. Watanabe, Y. Nogami, K. Oshima, H. Mori, S. Tanaka, J. Phys. Soc. Jpn. 68 (1999) 2654.

14) M. Watanabe, Y. Noda, Y. Nogami, H. Mori, J. Phys. Soc. Jpn. 73 (2004) 116; M. Watanabe, Y. Noda, Y. Nogami, H. Mori, J. Phys. Soc. Jpn. 74 (2005) 2011.

15) T. Mori, Bull. Chem. Soc. Jpn. 73 (2000) 2243.

16) R. Kondo, M. Higa, S. Kagoshima, H. Hoshino, T. Mori, H. Mori, J. Phys. Soc. Jpn. 75 (2006) 044716.

17) M. Kaneko, M. Ogata, J. Phys. Soc. Jpn. 75(2006) 014710. 